\title{
Pour un retour aux manuscrits de l'Arioste
}

\section{Simone Albonico}

\section{(2) OpenEdition}

\section{Journals}

Édition électronique

URL : https://journals.openedition.org/genesis/4446

DOI : 10.4000/genesis.4446

ISSN : 2268-1590

\section{Éditeur :}

Presses universitaires de Paris Sorbonne (PUPS), Société internationale de génétique artistique littéraire et scientifique (SIGALES)

\section{Édition imprimée}

Date de publication : 15 décembre 2019

Pagination : $31-46$

ISBN : 979-10-231-0650-3

ISSN : $1167-5101$

\section{Référence électronique}

Simone Albonico, «Pour un retour aux manuscrits de l'Arioste », Genesis [En ligne], 49 | 2019, mis en ligne le 01 décembre 2020, consulté le 12 janvier 2023. URL : http://journals.openedition.org/genesis/ 4446 ; DOI : https://doi.org/10.4000/genesis.4446 


\section{Pour un retour aux manuscrits de l'Arioste}

Simone Albonico

$\mathrm{L}$ es archives littéraires de Ludovico Ariosto (1474-1533) sont disparates et lacunaires ${ }^{1}$. Heureusement, le Roland furieux et les Satires font exception dans ce panorama, leur dossier comportant des matériaux originaux autographes d'un grand intérêt. Le cas du Roland furieux constitue, en particulier, un cas d'étude emblématique de la tradition ecdotique italienne, dans la mesure où la recension par Gianfranco Contini de l'édition des Frammenti autografi dell'Orlando furioso établie par Santorre Debenedetti en 1937 passe pour l'acte de naissance officiel de la «critique des variantes $»^{2}$. Nous nous proposons dans le présent article d'offrir une réflexion sur l'histoire éditoriale des textes de l'Arioste, en les mettant en regard de la naissance et de la consolidation de la «philologie d'auteur». Après avoir rappelé les prémisses interprétatives qui ont fondé les dispositifs éditoriaux retenus, puis le contexte historique et culturel qui a déterminé la réception critique de ces éditions, nous nous concentrerons sur les conséquences de ces dispositifs, notamment en ce qui concerne l'interprétation des autographes de l'Arioste.

Les deux œuvres de l'Arioste que nous examinerons (le Roland furieux et les Satires) présentent des dossiers génétiques aux caractéristiques différentes. Dans le cas des Satires - sept épîtres en tercets enchaînés composées par l'Arioste entre 1517 et 1525 mais jamais achevées ni publiées par lui comme un tout structuré -, nous disposons, en plus de trois témoins partiels, d'un manuscrit allographe actuellement conservé à Ferrare ${ }^{3}$, sur lequel l'auteur intervint pour introduire quelques corrections, de manière non systématique et incomplète. Le cas du Roland furieux est bien différent : l'auteur en procura de son vivant trois éditions imprimées, parues sous son contrôle en 1516, 1521 et 1532 (et siglées aujourd'hui A, B et $\mathrm{C}^{4}$ ), qui présentent des variantes et des modifications structurelles. Nous disposons en outre de ce que l'on nomme les Frammenti autografi dell'Orlando furioso ${ }^{5}$, qui attestent, bien que partiellement, du travail de réécriture intervenu entre la deuxième $(\mathrm{B})$ et la dernière édition procurée par l'auteur $(\mathrm{C})$, au cours duquel le poème passa de 40 à 46 chants. Les Frammenti autografi ne documentent pas

1. Pour un tableau d'ensemble, voir A. Casadei, «La tradizione delle opere di Ariosto », dans La tradizione dei testi, Rome, Salerno, «Storia della letteratura italiana», 10, 2001, p. 817-831.

2. Voir dans le présent numéro l'enjeu de Paola Italia, aux p. 45-57.

3. Ferrare, Biblioteca Comunale Ariostea, Cl. I, B, It. 2.

4. Il s'agit de : (A) Orlando furioso de Ludovico Ariosto da Ferrara, Ferrare, G. Mazzocco dal Bondeno, 1516; (B) Orlando furioso di Ludovico Ariosto ristampato et con molta diligentia da lui corretto, Ferrare, G. B. Pigna, 1521 ; et (C) Orlando furioso di messer Ludovico Ariosto nobile ferrarese. Nuovamente da lui proprio corretto e d'altri canti nuovi ampliato, Ferrare, F. Rosso da Valenza, 1532. L'édition critique de référence est celle de S. Debenedetti et C. Segre (éd.), Orlando furioso secondo l'edizione del 1532 con le varianti delle edizioni del 1516 e del 1521, Bologne, Commissione per i testi di lingua, 1960.

5. C'est ainsi que s'intitule l'édition fac-similée du manuscrit de Ferrare établie par G. Agnelli (éd.), I frammenti autografi dell'Orlando Furioso, Rome, Danesi, 1904. Nous nous référerons toujours dans ce texte à l'édition critique établie par S. Debenedetti (éd.), I frammenti autografi dell'Orlando furioso, Turin, Chiantore, « Giornale storico della letteratura italiana. Testi inediti o rari »1, 1937, reproduite en édition fac-similée (mais sans la page de titre originale), avec un avant-propos de C. Segre, Rome, Edizioni di Storia e Letteratura, 2010. 
l'ensemble des six chants ajoutés par l'Arioste et ne portent pas la trace de toutes les étapes de l'élaboration : ils constituent cependant un témoignage exceptionnel permettant de suivre une partie considérable de l'élaboration d'un des grands chefs-d'œuvre de la littérature occidentale. Néanmoins, force est de constater que les analyses philologiques et critiques ont porté presque exclusivement sur l'étude des variantes des éditions imprimées qui, certes, sont plus facilement accessibles aux spécialistes mais dont l'exploitation n'est pas sans comporter des difficultés; elles ont en revanche négligé les Frammenti, à savoir les documents du processus rédactionnel qui se prêtent pourtant davantage à une étude génétique. Il ne faut pas oublier enfin que le dossier génétique du poème, pour étoffé qu'il soit, reste très incomplet et qu'il est entièrement dépourvu, par exemple, d'indications méta-scripturales. Nous ne possédons aucun des matériaux relatifs à la première rédaction qui aboutit à l'édition princeps de 1516 (A) et dont l'Arioste parle dans une lettre célèbre adressée à Francesco Gonzaga le 14 juillet $1512^{6}$. Nous ne disposons pas non plus de l'exemplaire de l'édition de 1521 (B), sur lequel il corrigea le texte du poème en vue de la préparation de la dernière édition de 1532 (C), et qui est donc complémentaire de la rédaction des épisodes ajoutés : cet exemplaire, étudié et illustré au XvIe siècle par Giovan Battista Pigna (1529-1575), qui l'avait reçu du fils du poète, Virginio ${ }^{7}$, est aujourd'hui perdu. Rien ne reste, enfin, du travail de finition mené sur les épreuves de $\mathrm{C}^{8}$. Même les Frammenti autografi ne documentent qu'une petite portion de la dernière phase du travail rédactionnel. Le caractère lacunaire du dossier génétique du poème invite donc à la plus grande prudence lorsqu'il s'agit d'interpréter les matériaux disponibles. Ces derniers doivent être insérés dans un contexte dont nous ne pouvons reconstituer l'exact périmètre que par voie hypothétique. Si l'on excepte quelques feuillets où le texte prend forme pour la première fois (fig. 1), et d'autres où il est amplement retravaillé au fil de l'écriture, nous disposons pour l'essentiel de rédactions déjà bien abouties : la logique des corrections, parfois complexe, n'a pas encore été suffisamment analysée et expliquée, et elle ne concerne qu'en partie la véritable naissance de l'œuvre, témoignant dans une large mesure plutôt de sa réélaboration à travers un ensemble cohérent de réécritures successives.

\section{L'Arioste et la naissance de la critique des variantes}

Le développement des études philologiques portant sur l'Arioste permet de saisir un trait distinctif de la tradition italienne : l'histoire parallèle, certes entrelacée mais sans rapport de subordination, des techniques d'édition des manuscrits d'auteur (que l'on a par la suite nommées philologie d'auteur ${ }^{9}$ ) et des développements au $\mathrm{xx}^{\mathrm{e}}$ siècle de la critique des variantes, qui fut théorisée pour la première fois par Gianfranco Contini précisément en relation avec le cas de l'Arioste. Contini lui-même, fondateur et principal représentant de cette approche critique, a manifesté un certain désintérêt pour les solutions éditoriales

6. C'est ainsi qu'il fait référence au manuscrit original du Roland furieux auquel il travaille en vue de la première édition de 1516 : «Mais outre que le livre n’est ni limé ni encore achevé, comme tout ce qui est grand et qui nécessite du grand œuvre, il est aussi écrit avec tant de gloses et de ratures, et transporté de ci et de là, qu'il serait impossible à tout autre que moi de le lire. » (L. Ariosto, Lettere, éd. A. Stella, dans C. Segre (dir.), Tutte le opere di Ludovico Ariosto, vol. III. Satire Erbolato Lettere, Milan, Mondadori, 1984, p. 151, nous traduisons).

7. G. B. Pigna, I romanzi [...] al s. donno Luigi da Este [...] divisi in tre libri. Ne quali della poesia, et della vita dell'Ariosto con nuovo modo si tratta, Venise, Valgrisi, 1554, p. 65 et 123.

8. Outre S. Debenedetti, voir C. Fahy, L' «Orlando furioso» del 1532. Profilo di un'edizione, Milan, Vita e Pensiero, 1989 ; L. Spagnolo, «Variantistica ed ecdotica dell'Orlando furioso », Tipofilologia, 1, 2008, p. 61-87, notamment p. 66-71.

9. La formule est de Dante Isella : voir P. Italia, G. Raboni, Che cos'è la filologia d'autore, Rome, Carocci, 2010. 


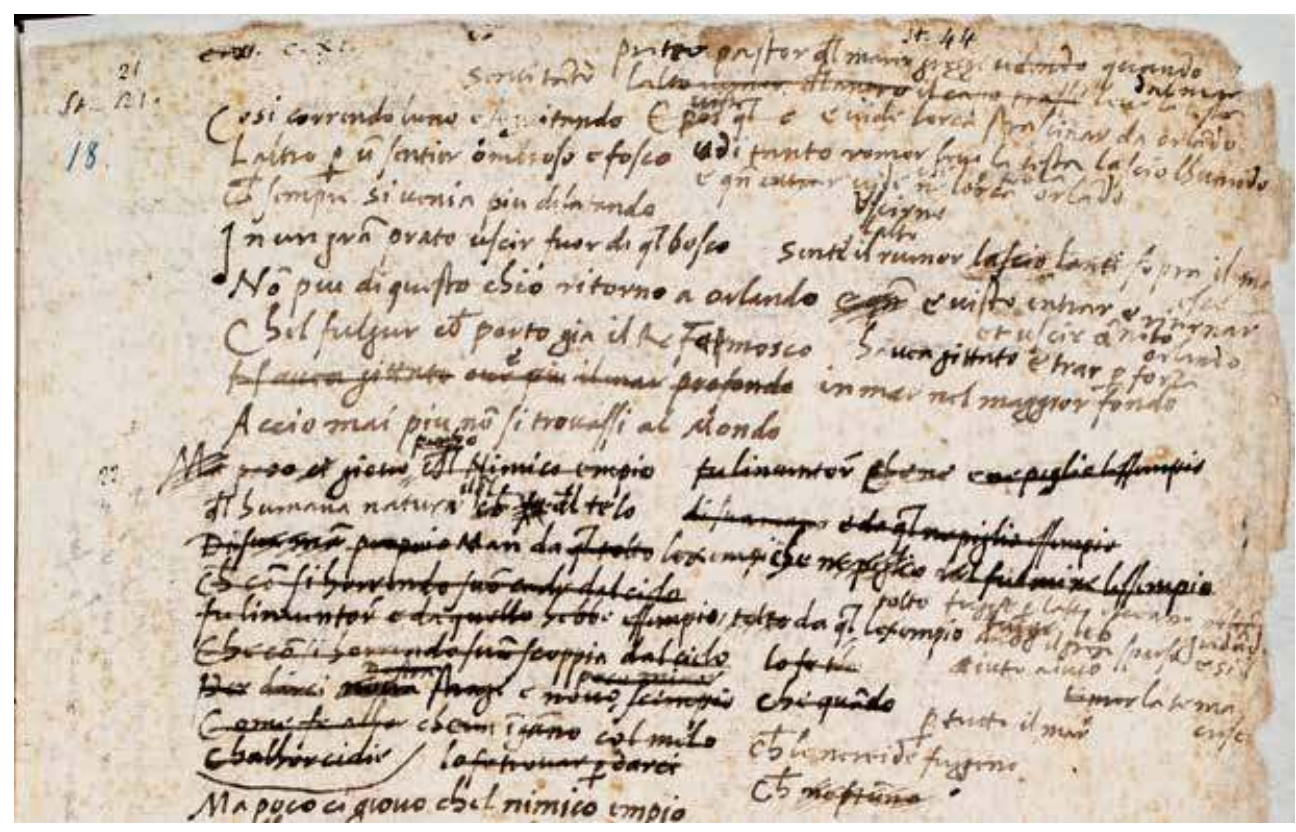

Fig. 1 : Ferrare, Biblioteca Comunale Ariostea, ms. Cl. I, A, It. 1, f. 18 r $^{\circ}$, moitié supérieure (la page entière mesure $320 \times 225 \mathrm{~mm}$ env.). Une fois épuisée la place disponible sur le f. $19 \mathrm{v}^{\circ}$, où il avait ébauché sans la finir la strophe 43 du chant XI, l'Arioste, qui travaillait «en aparté» sur ces deux feuilles, continue l'ébauche de ce passage en entamant une nouvelle strophe (la 44) sur la $\mathrm{f} .18 \mathrm{r}^{\circ}$, où il lui restait encore un peu de place. Le sujet mythologique de la strophe 43 (Ino et Mélicerte) servira pour la strophe 45, qui n’apparaît pas ici. Les numérotations des strophes sont de la main de Giovanni Andrea Barotti ou d'autres bibliothécaires.

(C) Ferrare, Biblioteca Comunale Ariostea.

Avec l'aimable autorisation du Ministero dei beni e delle attività culturali e del turismo. Reproduction interdite

et pour le problème de la «représentation » des manuscrits originaux, thèmes qui furent au contraire au cœur des remarquables recherches de son infatigable élève Dante Isella. C'est pourquoi les pages qui suivent tiendront compte de l'histoire entremêlée de ces deux traditions au long cours, philologico-éditoriale, d'un côté, et critique, de l'autre. De ce point de vue, la situation italienne se distingue nettement de la situation française pour deux raisons principales ${ }^{10}$. La première raison est la conservation de dossiers génétiques très anciens et d'une importance majeure dans la culture européenne (à partir de la production de Pétrarque en langue vulgaire et en latin), sur lesquels l'activité éditoriale et l'interprétation critique se sont exercées en parallèle depuis plus de cinq siècles : il ne s'agit donc pas en Italie d'un secteur d'études particulièrement «moderne». La seconde raison, qui n'est pas sans rapport avec cette première spécificité, tient à la persistance, tout au long de l'époque contemporaine, d'un intérêt pour la philologie reconstructive ou lachmannienne, indissociable d'un intérêt pour la philologie des originaux (pensons aux travaux de Michele Barbi, ou encore à Cesare Segre éditeur des Satires de l'Arioste) ${ }^{11}$.

Les différentes célébrations organisées en 1933 à l'occasion des quatre cents ans de la mort de l'Arioste permettent de cerner l'arrière-plan historique et culturel dans lequel s'inscrivent, peu après, l'édition des Frammenti par Santorre Debenedetti et le compte rendu qu'en fit Gianfranco Contini12. Alors que la promulgation des lois raciales, en 1938, allait

10. Voir 1'enjeu de Christian Del Vento dans le présent numéro, aux p. 13-27.

11. Voir G. Mazzocchi, «Filología de autor entre historia e método », AIEMH. Revista de la Asociación Internacional para el Estudio de Manuscritos Hispánicos, 1, 2015, p. 15-33.

12. G. Contini, «Come lavorava l'Ariosto », Meridiano di Roma, 18 juillet 1937, p. IV ; rééd. Id., Esercizi di lettura sopra autori contemporanei con un'appendice su testi non contemporanei, Florence, Parenti, 1939; Florence, Le Monnier, 1947; Turin, Einaudi, 1974 (édition citée). 
confiner l'édition de Debenedetti, qui était juif, dans une sorte d'espace insonorisé qui en compromit la fortune et la réception ${ }^{13}$, le compte rendu engagé et brillant de Contini avait inscrit cette édition exemplaire dans le panorama le plus avancé des études sur l'Arioste, notamment dans la droite ligne de l'approche critique, alors triomphante, de Benedetto Croce et de sa célèbre étude consacrée en 1917 à l'auteur du Roland furieux ${ }^{14}$. C'est là un aspect à certains égards paradoxal du texte de Contini - et il convient de le souligner d'emblée - si l'on compare le profil intellectuel de Debenedetti à celui de Croce et la nature très technique de son introduction, tout à la fois descriptive et reconstructive, qui verse, lorsqu'elle s'avance sur le terrain de l'interprétation critique, dans des considérations plutôt génériques ${ }^{15}$.

Deux raisons, complexes, pouvaient avoir poussé Contini à valoriser l'édition des Frammenti. Il entendait, en premier lieu, distinguer Debenedetti dans le domaine des études sur l'Arioste qui s'étaient toujours un peu cantonnées au répertoire de la célébration, même en dehors des manifestations commémoratives, et tendaient à faire un usage principalement ornemental des manuscrits originaux, dans des monographies ou des biographies certes de grande valeur mais souvent enclines à dépeindre l'atmosphère de la Renaissance et le clair-obscur de la vie de cour ${ }^{16}$. L'approche critique érudite prônée par l' "École historique» (qui avait dominé les études littéraires italiennes à la fin du XIXe et au début du XXe siècle et en laquelle Debenedetti lui-même, directeur du prestigieux Giornale storico della letteratura italiana, aimait à se reconnaître, quoiqu'on ne pût l'y réduire) avait certes produit d'excellents résultats ${ }^{17}$; mais l'édition des Frammenti était d'une teneur et d'une portée bien différentes : Contini entendait assurément, en soulignant les éléments de nouveauté qui la caractérisaient, affranchir Debenedetti, en qui il reconnaissait son maître ${ }^{18}$, des travaux qui l'avaient précédé, trop enclins à l'érudition, aux évocations, aux célébrations voire aux divagations 19 .

En deuxième lieu, le jeune critique tentait (avec une certaine hardiesse, il faut bien le reconnaître) de protéger l'œuvre de Debenedetti contre la réaction que menaçait de susciter son vertigineux «excès » de philologie, bien éloigné de la poétique de Croce alors prédominante.

13. Voir C. Segre, «Premessa», dans S. Debenedetti (éd.), I frammenti, 2010, op. cit., p. VIII.

14. B. Croce, «Ariosto» (1917), dans Id., Ariosto, Shakespeare e Corneille, Bari, Laterza, 1920, p. 1-70.

15. Voir notamment : «la str. 73, qui jaillit avec une immédiate limpidité»; «Les vers et demi-vers s'écoulent, s'insinuent [...] comme une eau débordante. La force est telle que [...]»; «Ébauches composées fiévreusement»; «accès de grandeur humaine, qui recevront dans les révisions suivantes, comme des épreuves d'eaux-fortes, plus de lumière et de relief »; «Mais ce que ces feuilles ont de plus précieux, joyaux rares ou uniques, sont ces ébauches jetées en marge par une main fébrile»... (S. Debenedetti [éd.], Iframmenti, 2010, op. cit., p. XXIII). Nous traduisons. 16. Même pour les plus méritantes, comme celle de M. Catalano, Vita di Ludovico Ariosto : ricostruita su nuovi documenti, Genève, L.S. Olschki, 1930-1931.

17. Outre la biographie de M. Catalano, accompagnée d'un important volume de documents (auquel avait ouvert la voie en 1926 un premier recueil préparé dès 1901 par G. Sforza, Documenti inediti per servire alla vita di Ludovico Ariosto raccolti e illustrati, Modène, Società Tipografica moderna, 1926), mentionnons au moins les Annali delle edizioni ariostee (2 vol., Bologne, Zanichelli) et le catalogue des manuscrits de la Biblioteca Comunale Ariostea (vol. LIV des Inventari dei manoscritti delle biblioteche d'Italia, Florence, Olschki), édités tous deux par G. Agnelli et G. Ravegnani en 1933.

18. Voir au moins C. Segre, «Santorre Debenedetti nella filologia di Gianfranco Contini » (1999), dans Id., Dai metodi ai testi. Varianti, personaggi, narrazioni, Turin, Aragno, 2008, p. 89-106.

19. 1933 est aussi l'année du volume collectif très inégal dirigé par Antonio Baldini, L'ottava d'oro : la vita e l'opera di Ludovico Ariosto. Letture tenute in Ferrara per il quarto centenario dalla morte del poeta, Milan, Mondadori, 1933, précédé de «Due messaggi di Gabriele D’Annunzio». 


\begin{tabular}{|c|c|c|c|c|}
\hline \multirow{2}{*}{$\begin{array}{l}\text { Ajouts de l'édition C } \\
\text { (1532) }\end{array}$} & \multicolumn{4}{|c|}{ Textes présents dans les Frammenti } \\
\hline & $\begin{array}{l}\text { "mala copia" } \\
\text { (ébauche) }\end{array}$ & $\begin{array}{l}\text { "brutta copia" } \\
\text { (brouillon) }\end{array}$ & $\begin{array}{l}\text { "buona copia" } \\
\text { (mise au net) }\end{array}$ & $\begin{array}{l}\text { "copia definitiva" } \\
\text { (copie définitive) }\end{array}$ \\
\hline \multicolumn{5}{|l|}{ Olympie } \\
\hline & $18-19$ & FASC. I & & FASC. II-IV \\
\hline IX 8-94 & & $8-39,83-94$ & & $1-94$ \\
\hline$X 1-34$ & & $1-18$ & & $1-35$ \\
\hline XI 21-80 & $21-44,71,73$ & & & $21-70$ \\
\hline XII 1-4, 17 & 1 & & & $8-17$ \\
\hline \multicolumn{5}{|l|}{ Marganor } \\
\hline & & FASC. V & & \\
\hline$X X X V I 84, X X X V I I$ 1-122 & & $25-122$ & & \\
\hline \multicolumn{5}{|l|}{ Roger et Léon } \\
\hline & & & FASC. VI-VII & \\
\hline XLIV 12-14, 36-104 & & & $11-20,31-92,104$ & \\
\hline$X L V 1-117$ & & & $1-3,7-117$ & \\
\hline XLVI 19-66, 69-72 & & & $50-71$ & \\
\hline
\end{tabular}

Fig. 2 : Représentation schématique du contenu et de la disposition des épisodes présents dans les Frammenti autografi dell'Orlando Furioso. Dans la première colonne figurent les parties ajoutées dans l'édition C du Roland furieux (1532). Dans les colonnes suivantes sont signalés les textes présents dans les Frammenti, rangés de gauche à droite selon leur degré d'achèvement établi par Debenedetti (nous reprenons cette disposition par simplicité même si elle peut soulever des objections).

À cette fin, Contini reformulait en son nom propre une charge critique qui n'était que sousjacente dans le texte de Debenedetti, et, suivant son goût, la synthétisait et lui attribuait une dimension fortement novatrice (sur le plan général de la méthode, bien plus que sur celui de l'interprétation de l'Arioste). Ce faisant, Contini, du moins aux yeux de ses lecteurs, infiniment plus nombreux que ceux de l'édition de 1937 des Frammenti, transformait cette dernière en quelque chose de bien différent de ce qu'elle se proposait d'être 20 .

L'édition des Frammenti autografi s'appuie sur le manuscrit autographe actuellement conservé à Ferrare (Biblioteca Comunale Ariostea, siglé Cl. I, A, It. 1), complété par des documents aujourd'hui conservés à Naples et à la Bibliothèque Ambrosienne de Milan21. Il témoigne de l'élaboration de trois épisodes du poème (celui d'Olympie, celui de Marganorre et celui de Roger et Léon, voir fig. 2), dont le récit scindé en plusieurs groupes d'octaves est réparti entre divers chants du poème, selon la technique de l'entrelacement qui caractérise la composition du Roland furieux et qui donne lieu à d'incessantes interruptions du fil narratif, abandonné puis repris par intermittence.

20. Voir C. Segre, «Premessa», dans S. Debenedetti (éd.), I frammenti, 2010, op. cit., p. VIII-IX.

21. Le manuscrit H 55 inf. de la Bibliothèque Ambrosienne de Milan conserve des éléments apographes relatifs à l'épisode du château de la Roche-Tristan (XxxII, 76-81 et 97-109), présent dans l'édition C, et à vingt octaves abandonnées (dites «de l'Histoire d'Italie»). Le manuscrit San Martino 353 de la Bibliothèque nationale de Naples contient quinze autres octaves abandonnées mais autographes («l'Écu de la reine Élise»). 
L'édition des Frammenti avait plusieurs mérites et qualités : elle parvenait de façon magistrale à déchiffrer la graphie de l'Arioste, à dénicher les premières leçons enfouies sous les corrections, à situer à leur juste place les fragments de Milan et de Naples grâce à sa reconstitution codicologique, à établir la chronologie des interventions de l'Arioste et à représenter les étapes de l'élaboration du texte avec clarté et esprit de synthèse. Bien des vertus de ce travail sont toutefois restées dans l'ombre, non seulement - ou plutôt non pas tant - en raison du succès de la recension de Contini, qui n'insistait volontairement que sur certains aspects de l'édition des Frammenti, mais bien davantage en raison du silence qui entoura par la suite les autographes et leur édition, un silence qui dura - en substance - jusqu'à aujourd'hui.

La solution que Debenedetti adopta pour représenter la situation des autographes répondait à un critère fondamentalement mimétique, en fonction duquel la disposition des octaves sur la page et celle des corrections s'efforçaient de reproduire la topographie des autographes (fig. 3). Cependant, Debenedetti insistait à raison sur la nature critique et non diplomatique de son édition, grâce à laquelle il avait été en mesure de reconnaître les erreurs de l'Arioste, de les signaler et de les corriger, en restaurant des lettres oubliées ou interverties, des syllabes, des mots ou des vers anticipés, oubliés ou répétés, des corrections incomplètes, des étourderies diverses, sans oublier la ponctuation et les majuscules. La démarche mimétique qu'il avait adoptée n'avait que très peu cours dans la tradition moderne, principalement pour de banales (mais décisives) raisons techniques et économiques. En outre, l'éditeur ne prétendait pas par ce choix faire preuve d'une absolue originalité, comme l'a récemment expliqué Cesare Segre 22 : il était plutôt mû par la volonté de réactualiser, ou plus simplement de prolonger une tradition qui remontait à la solution expérimentée par Federico Ubaldini dans son édition des brouillons de Pétrarque (le manuscrit Vatican Latin 3196), parue à Rome en 1642. L'alternance entre caractères romains et italiques, le recours à des caractères de différentes tailles, la dislocation mimétique du texte sur la page, quelques rares symboles éditoriaux sont exactement les mêmes outils que ceux utilisés par Ubaldini dans son édition génétique «avant la lettre» des brouillons de Pétrarque. Debenedetti s'en servit en faisant appel à très peu de formalisations graphiques supplémentaires et en s'appuyant sur un appareil de notes particulièrement réduit (fig. 4).

Je ne crois pas cependant que l'unique raison de la maigre fortune de ce système de représentation éditoriale soit liée à ses coûts (même s'il s'agit là d'une composante souvent négligée et pourtant non moins essentielle dans l'histoire de la philologie que dans l'histoire de l'édition). Au Xxe siècle et jusqu'à une époque récente, la tradition philologique italienne semble dominée, pour ne pas dire bloquée, par son attachement à une sorte de parfaite autonomie du «texte», dont elle préserve l'unité et la continuité en reléguant l'apparat critique dont il est parfois accompagné dans des zones marginales et bien distinctes. C'est ainsi que la philologie d'auteur conserve un goût pour l'organisation du texte et pour la clarté de la page imprimée qu'on ne peut certes pas imputer à Croce mais qui ne répond pas moins pleinement

22. Voir C. Segre, «Petrarca e gl'incunaboli della critica genetica» (2003), dans Id., Dai metodi ai testi, op. cit., p. 159-164; Id., «Premessa», dans S. Debenedetti (éd.), I frammenti, 2010, op. cit., p. VI. Voir aussi P. Italia, «Alle origini della filologia d'autore», dans C. Caruso, E. Russo (dir.), La filologia in Italia nel Rinascimento, Rome, Edizioni di Storia e Letteratura, 2018, p. 379-398. 
aux attentes et aux idiosyncrasies de ce dernier. Le texte, même lorsqu'il représente une situation documentaire complexe et stratifiée, conserve un haut niveau d'abstraction. Il faut souligner le silence absolu de Contini sur un élément essentiel de l'édition de Debenedetti qui contribue, plus que toute autre, à la présentation claire et efficace du texte : à savoir le système de représentation adopté, mimético-reproductif, qui était intrinsèquement contraire aux principes de Croce et qui nous permet, aujourd'hui encore, de suivre aisément les circonvolutions parfois obscures du processus créatif de l'Arioste. On saisit là l'une des spécificités de la position de Contini : il accorde une grande attention aux variantes pouvant donner prise à l'élaboration d'un discours critique général (bientôt étoffé par ses essais sur Pétrarque et Leopardi23), mais, ici comme ailleurs, il se désintéresse fondamentalement des aspects «matériels» du texte manuscrit ainsi que des détails de sa restitution éditoriale (point essentiel qu'ont peut-être négligé les générations qui se sont consacrées par la suite à ce domaine d'études). Cet élément permet de mesurer l'assise pour ainsi dire «crocienne» de la philologie des originaux propre à Contini, qui débouche précisément sur une pure critique : la critique des variantes.

De tels postulats, de telles tendances, une telle prudence, expliquent probablement pourquoi Contini exclut de sa réflexion critique - et considère comme irrecevables - les corrections de l'Arioste dont il estime, dans un schéma de pensée tout «crocien», qu'elles relèvent du passage «du non-être à l'être poétique». Son attention se porte exclusivement sur les interventions du poète qui remplacent des portions du texte «fragmentairement valides » par d'autres « organiquement valides », c'est-à-dire sur les variantes substitutives, considérées dans une perspective de perfectionnement du texte ${ }^{24}$. Les cas où se manifeste la lente et parfois laborieuse mise au point d'une idée poétique, ou encore de substantiels changements de perspective, en un mot l'inventio en acte, sont certes d'un immense intérêt, mais ils s'avéraient impropres ou négligeables pour l'esthétique diffuse de l'époque (et par la suite dans une Italie qui resta longtemps et souvent inconsciemment «crocienne»); ils se montraient en outre difficiles à exploiter au regard du type de publication choisi pour mener la discussion : l'hebdomadaire culturel Meridiano di Roma, qui assurait une bonne audience à l'essai de Contini mais qui n'était pas le lieu propice à un véritable développement de son raisonnement 25 .

Dans la récente (2010) réimpression de l'édition de 1937, Cesare Segre attribue l'oubli qui frappa ce texte à la dramatique situation historique engendrée par les lois raciales promulguées en 1938. Je ne partage qu'en partie cette analyse, d'autant plus que Segre, neveu de

23. Aujourd'hui réunis avec d'autres essais dans G. Contini, Varianti e altra linguistica. Una raccolta di saggi (1938-1968), Turin, Einaudi, 1970.

24. Voir dans le présent numéro l'enjeu de Paola Italia, ainsi que la reconstruction proposée par M. T. Giaveri, «La critique génétique en Italie : Contini, Croce et "l'étude des paperasses" », Genesis, n 3, 1993, p. 9-29, notamment p. 14 et 19-22; C. Segre, «Critique des variantes et critique génétique», Genesis, n 7, 1995, p. 29-45, notamment p. 36 et $44-45$.

25. Sur Contini et Croce, outre les travaux importants de G. Lucchini (réunis dans Studi su Gianfranco Contini : «fra laboratorio e letteratura». Dalla critica stilistica alla grammatica della poesia, Pise, ETS, 2011), de C. Segre (Contini, Croce e la critica degli scartafacci, Florence, SISMEL, 2004) et de M. Ciliberto (L'influenza culturale di Benedetto Croce, intr. de M. Ciliberto, Pise, Ed. della Normale, 2013), voir notamment F. Finotti, «La storia finita. Filologia e critica degli scartafacci», Lettere italiane, 46, 1994, p. 3-43. 
C. XI.

[2I]

Cosi correndo l'uno, e seguitando

L'altro, per un sentier ombroso e fosco

Che sempre si venfa piú dilatando,

In un gran prato uscir fuor di quei bosco.

Non piú di questo; ch'io ritorno a Orlando.

Che '1 fulgur che port' già il re Fermosco.

Havea gittato ove piú il mar profondo,

Acció mai piú non si trovassi al mondo.

[22]

Ma poco ci giovd: che 'l nimico empio

De l'humana natura, che fe' il telo

Di sua man [Di] propria man, da quel tolto l'exempio, Di sua mano, e da quel ne piglid essempio, Che con si horrendo suon cade dal cielo

[Poco gioud: perhd che 'l nimico empio De l'humana natura], il qual del [telo] Fu l'inventore, e da quello hebbe essempio,

Che con si horrendo suon scoppia dal cielo;

Per darci nova strage e novo scempio.

Che quando Chilior ci did.

Come fo' alhor ch'Eva ingannd col melo,

Lo fe' trovar por darci

Ma poco ci giovò: che '1 nimico empio

De l'humana natura, il qual del telo

Fu l'inventore, havendone l'essempio

Preso da quel che ribombar ta il cielo;

Con forse non minor di quello scempio

Che ci die quando Eva inganno col melo,

Lo fe' trova Lo fece ritrovar da un negromante Lo fece

Al tempo de' nostri avi o poco inante. internal,
La machina crudel, di piú di cento
ove

Passa d'aqua $\times$ stè ascosa molt'anni,

Al sommo tratta per incantamento,

Prima

Portata in prima fu tra gli Alamanni;

Che fatto $\mathrm{Li}$ quali uno et un altro experimento

Facendo, e procurando i nostri danni

Feceno e tanti

Facendone, e il demonio a' nostri danni

Assuttigliando lor via piú la mente,

$N^{\prime}$ han ritrovato l'uso finalmente.

Procu Procacciar non cessando i proprij danni

Ne ritrovaro
-Fu l'inventor, che ne

[Ful'inventor]e, e ne piglid l'essempio

[Fu l'inventor], tolto da quel l'exempio,

[Fu l'inventor], c'hebbe da quel l'essempio, Ch'apre le nube $\mathrm{e}$ in terra vien da cielo:

22 (prima redazione), 2, il tejo. L'articolo è coperto da del della seconda redazione.

- (seconda redaziono), 7. Lo fe' trovar per darci. Nel ms., Lo fé tro d̀ ancora ripetuto, e cancellato, accanto a! v. 4 .

- (ultima redazione). 4. da cjolo. La $c$ pare copra una $l$ incompiuta (dal?).

23. 5. experimento. Seguouo due lettere cancellate.
N. B. - Nell'angolo superiore destro di c. 18 r. l'A. abbozza la st. 44 di questo canto (v. p. 28).

Fig. 3 : S. Debenedetti (éd.), I frammenti autografi dell'Orlando furioso, Turin, Chiantore, 1937, p. 21, chant XI, strophes 21-23, tirées du f. 18 r $^{\circ}$ du ms. de Ferrare des Frammenti. 


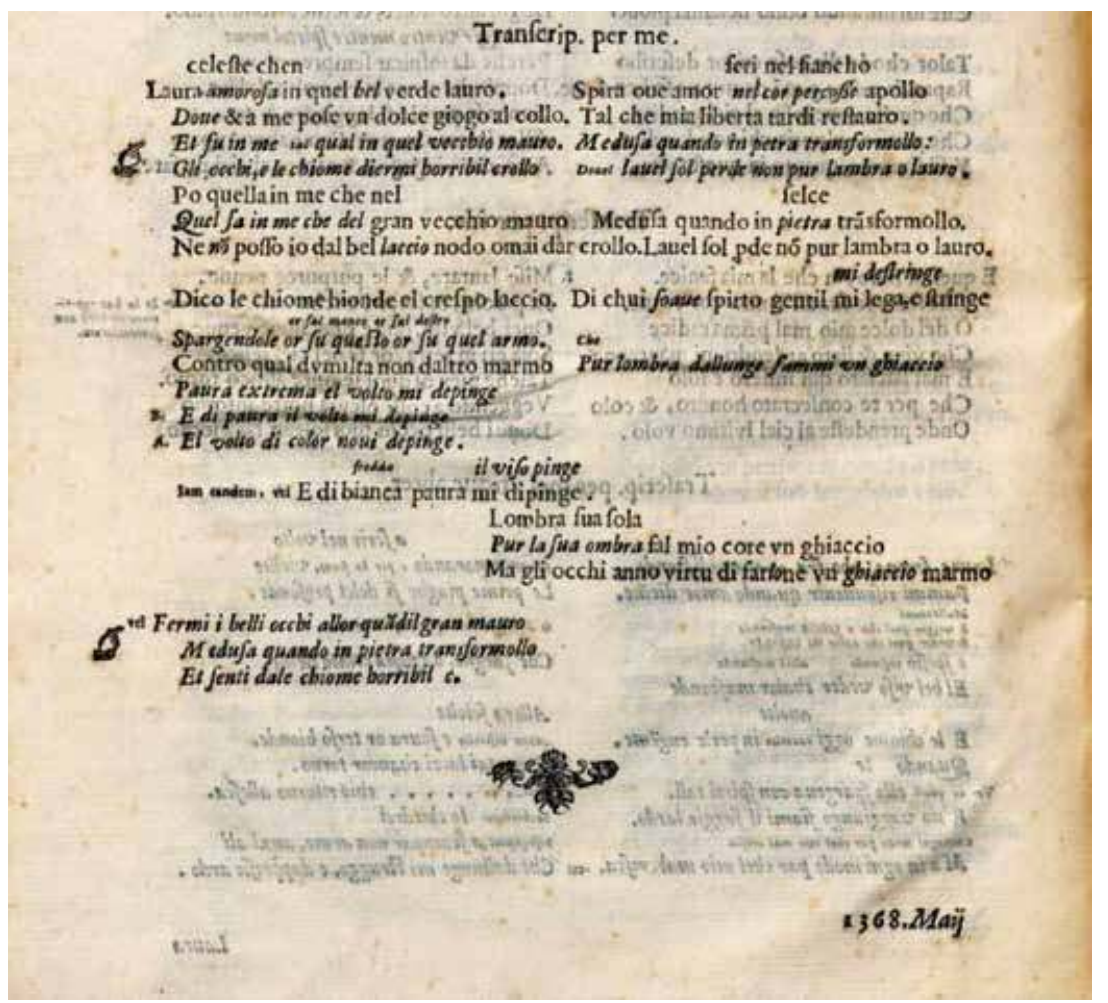

Fig. 4 : Le Rime di F. Petrarca estratte da un suo originale [...], [éd. Federico Ubaldini], Rome, Stamperia del Grignani, 1642, p. IV. Détail de la page où sont édités les deux derniers textes du folio $2 \mathrm{r}^{\circ}$ du ms. Vatican Latin 3196 de la Bibliothèque Apostolique Vaticane (Codice degli abbozzi); nous reproduisons la moitié inférieure de la page, relative au sonnet 197 du Chansonnier (Rerum vulgarium fragmenta).

Debenedetti, ne s'était pas non plus particulièrement consacré aux textes édités par son oncle : il en fit assurément un usage répété au cours de son intense activité de spécialiste reconnu de l'Arioste, mais presque exclusivement pour dater des phénomènes linguistiques. La conjuration du silence est d'autant plus surprenante qu'au cours du $\mathrm{Xx}^{\mathrm{e}}$ siècle la critique des variantes, et même la philologie d'auteur, ont trouvé dans l'article de Contini leur texte fondateur, sans que personne ne sente le besoin d'y revenir pour vérifier, d'un côté, le bien-fondé et la fiabilité de ce constat et pour approfondir, de l'autre, tous les aspects de l'inventio et de la création poétique de l'Arioste que Contini avait laissé de côté. Comme si la fortune du compte rendu avait enseveli les Frammenti et leur éditeur sous une chape d'oubli scientifique et critique. Dans l'article de Contini, comme l'a relevé Segre, prévaut une indéniable attention pour la méthode, qui s'accompagne d'un certain désintérêt pour les textes de l'Arioste; mais la culture philologique italienne, qui s'est longtemps abreuvée à cet article de façon tendanciellement acritique (et on ne peut certes pas en faire reproche à Contini), ne s'est pas rendu compte de l'écart qui séparait les intentions et les intérêts de l'éditeur et ceux de son jeune disciple. Si un usage «simplifié» de ces variantes a prévalu, il est sans doute difficile de considérer avec Segre que l'édition Debenedetti est «une édition génétique», qui «anticipe de quelques décennies la critique génétique française ${ }^{26}$. Ce fut autre chose : quelque chose «de plus », au sens où ce travail s'inscrivait dans un projet d'édition critique de toute l'histoire textuelle du Roland furieux, et quelque chose «de moins», au sens où il lui manquait une volonté interprétative explicite de l'avant-texte, qui fût au moins aussi affirmée que les préoccupations philologico-éditoriales.

26. C. Segre dans I frammenti autografi dell'Orlando furioso, éd. S. Debenedetti, Rome, Edizioni di Storia e Letteratura, 2010, p. VIII. 

Revenir aux manuscrits
de l'Arioste

S'il faut pleinement reconnaître les mérites de Debenedetti en ce qui concerne la représentation de la situation manuscrite et la restitution du texte de l'Arioste, il convient cependant de faire état des nombreux aspects des Frammenti qui, pour les diverses raisons que nous avons évoquées, sont restés vierges de toute analyse jusqu'à il y a peu. Une étude importante et très récente d'Ida Campeggiani 27 les a enfin affrontés. Ce n'est pas un hasard si l'intérêt de la chercheuse est avant tout critique et historique, avec un horizon très large. Par son titre même, l'ouvrage fait comprendre qu'il refuse de se cantonner à des problématiques strictement textuelles ou à des considérations monographiques, et cela montre bien que la philologie ne peut faire de progrès significatifs que si elle adopte des perspectives d'une certaine ampleur.

Repartons donc des manuscrits de l'Arioste édités par Debenedetti afin de formuler quelques considérations :

a) Debenedetti avance dans son «Introduction» certaines observations sur les feuilles 18-19 du manuscrit de Ferrare et offre des indications sur les rapports chronologiques qui unissent les épisodes ajoutés (en particulier l'épisode d'Olympie et celui de Marganor). Mais le critique a tendance à situer sur le même plan toutes les parties constitutives d'une même rédaction dès lors que n'apparaissent pas de signes manifestes d'une élaboration en cours (par exemple plusieurs réécritures inabouties d'un même segment) : il ne prend pas en compte les éventuelles relations à distance (pas nécessairement de type structurel) qui lient des épisodes différents ou des phases différentes de la rédaction d'un même épisode (dans cette perspective, il serait utile d'approfondir l'analyse de la variabilité du ductus et de la graphie).

Campeggiani, pour sa part, reprend toute la question 28 et parvient à restituer un tableau plus complexe (notamment en ce qui concerne les élaborations au fil de la plume pour les chants IX et XLV et les rapports existants entre les différents épisodes ajoutés) : elle a ainsi ouvert la voie à de nouvelles et fructueuses études.

b) Outre ces considérations philologiques, les manuscrits de Ferrare permettent aussi d'examiner le développement narratif et idéologique (historique, politique) de certains passages. Debenedetti n'avait certes pas négligé cet aspect, mais s'était limité à quelques exemples. Or, après lui, à l'exception d'un essai ancien d'Enrico Carrara sur l'épisode de Marganor, en 194029, et du livre de Campeggiani (qui se penche notamment sur la célèbre invective contre les armes à feu et sur le titre royal conféré à Roger), personne ne semble avoir abordé les Frammenti sous cet angle (que Contini mentionne en passant dans la première partie de son compte rendu ${ }^{30}$ ), dont il n'est pas exagéré de dire qu'il pourrait aujourd'hui constituer le cœur de toute analyse.

c) Debenedetti ne traita pas, du moins publiquement, les aspects linguistiques des Frammenti, tandis que Segre s'en servit comme point de référence pour reconstituer le développement de la langue littéraire de l'Arioste, documentée à des degrés divers, de manière non autographe, par les autres témoins du Roland furieux (les impressions A, B, C)

27. I. Campeggiani, L’ultimo Ariosto. Dalle «Satire» ai «Frammenti autografi», Pise, Ed. della Normale, 2017. 28. Ibid., p. 347-356 et 365-408.

29. E. Carrara, «Marganorre», Annali della Scuola Normale Superiore di Pisa. Lettere, série 2, 9, 1940, p. 1-20, et $155-182$.

30. G. Contini, «Come lavorava l'Ariosto», art. cit., p. 234. 
ou par d'autres œuvres (les Cinq chants, les Satires) ${ }^{31}$, sans toutefois mettre à disposition des dépouillements ou des études systématiques. Campeggiani a mis en évidence tout ce qui reste à faire aussi sur ce versant 32 . Les Frammenti, rappelons-le, préparent la troisième impression du poème (1532), dans laquelle l'Arioste revoit de façon substantielle l'aspect linguistique du Roland furieux en réponse aux théories énoncées par Pietro Bembo dans les Prose della volgar lingua $(1525)^{33}$. Campeggiani a remarqué pour la première fois que, dans ce qui devait être une copie mise au net de l'épisode de Roger et Léon, dans une version avancée donc, apparaissent des formes dialectales et des phénomènes d'hyper-correction que l'Arioste, en théorie, n'aurait plus dû commettre depuis bien longtemps. Cela montre combien notre approche de l'évolution linguistique de l'Arioste, fondée sur le préjugé d'un développement linéaire et cohérent, reste simplificatrice et appelle à être vérifiée très précisément, voire reconsidérée, avec tout ce que cela implique sur le plan éditorial (y compris pour les Satires).

d) Tant Debenedetti que Segre considéraient qu'il était inapproprié de représenter le texte des Frammenti dans une position subordonnée par rapport au texte $\mathrm{C}$, une solution éditoriale sans doute peu efficace. Cette conviction repose principalement sur l'idée d'une irréductible altérité entre, d'un côté, la phase créatrice et privée, et, de l'autre, le texte achevé et publiquement diffusé34 - distinction qui (quelle que fût son origine) s'accordait, au fond, avec l'idée épurée de «Texte» prônée par Croce. Il serait pourtant opportun, et simple, de proposer une comparaison directe entre les variantes du dernier état textuel des Frammenti et la version imprimée de 1532 (C), en ce qui concerne non seulement la dernière rédaction, très avancée, de l'épisode d'Olympie, mais aussi l'épisode de Roger et Léon 35.

Il importe surtout de mener de nouvelles analyses stylistiques qui corrigent celles de Contini. Prenons le cas du prétendu «anti-alexandrinisme» de l'Arioste. Il faut savoir qu'en italien la catégorie critique d' «alexandrinisme» désigne de façon positive la plus haute production poétique latine, de Catulle à Ovide, marquée par l'érudition, par la préciosité et le raffinement des images. Or Contini l'utilise pour sa part dans un sens péjoratif, en vertu d'une approche simplificatrice. Affirmant au terme de son article que «l'alexandrinisme est l'ennemi déclaré de l'Arioste ${ }^{36}$ », il estime que le poète aurait utilisé, dans son travail de correction, différents «procédés» visant à le « détruire 37 ». Son affirmation n'est étayée que par deux exemples et, surtout, par une référence à Benedetto Croce : ce dernier estimait en effet que l'Arioste, mû par une recherche de l'«Harmonie» - trait distinctif de sa poésie -,

31. C. Segre, «Studi sui "Cinque Canti”», dans Id., Esperienze ariostesche, Pise, Nistri-Lischi, 1966, p. 121-177, notamment p. 165-172; Id., «Storia testuale e linguistica delle "Satire” », dans Id. (dir.), Ludovico Ariosto : lingua, stile e tradizione, Milan, Feltrinelli, 1976, p. 315-330.

32. I. Campeggiani, L'ultimo Ariosto, op. cit., p. 384-408.

33. Voir la Présentation du présent numéro et l'enjeu de Christian Del Vento.

34. Cette hypothèse a été également proposée pour d'autres auteurs. Ainsi, dans son édition des Chants de Giacomo Leopardi (Milan, Il Polifilo, 1984), Domenico De Robertis distingue la tradition manuscrite des Canti et la tradition imprimée.

35. L. Spagnolo («Variantistica ed ecdotica dell'Orlando furioso», art. cit., p. 71-72) propose pour l'épisode d'Olympie de corriger le texte C en se fondant sur les Frammenti; sur l'épisode de Roger et Léon, voir I. Campeggiani, L'ultimo Ariosto, op. cit., p. 384-408.

36. G. Contini, «Come lavorava l'Ariosto», art. cit., p. 239.

37. Ibid., p. 240. 
avait eu recours au procédé de l'ironie dans l'intention d'en finir avec l'alexandrinisme. Fidèle à cette interprétation, Contini conclut sans surprise que «l'orientation profonde du travail de correction mené par l'Arioste correspond parfaitement à la description» qu'en donnait Benedetto Croce ${ }^{38}$. Il faut dire que Croce et Contini étaient peu sensibles, pour des raisons d'affinités culturelles, de goût mais aussi d'orientations esthétiques générales, au style précieux de l'alexandrinisme, poésie allusive et recherchée. Ce courant avait pourtant constitué, historiquement, la ligne de force d'une littérature dont le cadre dépassait la seule péninsule italienne : depuis l'Antiquité grecque et latine jusqu'à la Renaissance latine et volgare, puis jusqu'au classicisme de poètes tels que Vincenzo Monti (1754-1828), Ugo Foscolo (1778-1827), Giacomo Leopardi (1798-1837) et Giosuè Carducci (1835-1907), l'alexandrinisme avait même été au XIXe siècle le contemporain et le concurrent d'un romantisme moins vigoureux en Italie qu'ailleurs en Europe. Et c'est précisément dans cette longue lignée que s'inscrivaient le Roland furieux et les Satires : prenons-en pour preuve l'admiration de l'Arioste pour la poésie lyrique d'Horace et les nombreuses imitations de Virgile et d'Ovide que recèlent les vers de son poème épique. Il nous est donc impossible de partager la position de Contini, qui, loin de contredire l'idée de fond de Croce, tend au contraire à la valider. Son approche critique - qui fut certes en son temps «à la page» et qui eut le mérite d'enrichir l'horizon «crocien» de nouvelles techniques interprétatives - finit ainsi pas trahir l'Arioste, tant du point de vue de l'étude de ses manuscrits, de leur phénoménologie et de leur sens général, que du point de vue de leur interprétation littéraire.

\section{La tradition des Satires}

Dans cette dernière partie nous présenterons le second cas que nous nous sommes proposé d'aborder, celui des Satires. Comme nous le disions en ouverture, le dossier génétique de cette œuvre est constitué de trois témoins partiels et d'un allographe, F39, qui est l'œuvre d'un copiste dont on ne sait pas s'il fut vraiment fidèle aux détails de la langue originale de l'Arioste, qui révisa lui-même le manuscrit. L'analyse de la tradition philologique des Satires permet ainsi de formuler une série de considérations, dont certaines concernent le manuscrit $F$ et d'autres, en conséquence, les solutions retenues par Cesare Segre dans son édition qui fait aujourd'hui référence ${ }^{40}$. Ces deux niveaux sont étroitement imbriqués, confirmant qu'il est possible de séparer l'analyse des manuscrits et leur représentation éditoriale seulement dans une perspective historico-critique, celle-là même qui peut suggérer de nouvelles analyses, des choix différents et des interprétations renouvelées.

En 1875, parut une reproduction lithographique du manuscrit $\mathrm{F}^{41}$, dont on pensait alors qu'il était entièrement autographe. En 1903, toutefois, Giovanni Tambara démontra que la rédaction principale ne pouvait être attribuée à l'Arioste ${ }^{42}$ et le débat tourna longtemps autour de l'identité du scripteur de cette dernière, ainsi que des interventions marginales. Il fallut

\footnotetext{
38. Ibid., p. 241.

39. Ferrare, Biblioteca Comunale Ariostea, Cl. I, B, It. 2.

40. Parue d'abord chez Mondadori, en 1984 (L. Ariosto, Satire, éd. C. Segre, dans Id., Satire, Erbolato, Lettere, op. cit.), puis chez Einaudi, en 1987 (Id., Satire, éd. C. Segre, Turin, Einaudi, 1987).

41. Le Satire autografe di Ludovico Ariosto pubblicate a cura del Comitato ferrarese per la ricorrenza del IV centenario ariostesco, Bologne, Zanichelli e Romagnoli, 1875.

42. L. Ariosto, Satire, éd. G. Tambara, Livourne, Giusti, 1903.
} 
attendre les travaux de Carlo Bertani et de Michele Catalano 43 pour que soient correctement identifiées, d'une part, les corrections de la main du copiste et, de l'autre, les corrections apportées à plusieurs reprises par l'auteur, avec plusieurs encres différentes, parmi lesquelles prédominait un gris clair bien distinct. D'un point de vue éditorial, il en découla l'idée, dont s'empara ensuite Debenedetti ${ }^{44}$, que toutes les corrections avaient vocation à être reproduites, et que même celles qui n'étaient pas autographes, à de très rares exceptions, répondaient à la volonté de l'auteur.

Segre, après avoir publié en 1954 le texte critique précédemment préparé par Debenedetti, mena dans les années 1970 (à l'approche de nouvelles commémorations) des recherches en vue de l'édition critique, mais en procédant différemment. Tandis que les études précédentes avaient reconnu la présence de la main de l'auteur sur le manuscrit $\mathrm{F}$ à partir d'une enquête paléographique (graphie et encre), Segre analysa les interventions marginales selon des critères avant tout linguistiques. Son projet n'était pas tant d'attester leur caractère autographe, ce qu'il est impossible de faire avec une absolue certitude, que l' «authenticité » des formes, c'est-à-dire la correspondance (ou non) de ces dernières avec ce que l'on connaît des usages linguistiques de l'Arioste. Ces analyses se fondaient sur l'évolution de la langue du Roland furieux que Segre lui-même avait synthétiquement tracée à partir des trois éditions originales (avec une série de corrections apportées à la troisième, pour l'édition critique de 1960) et des Frammenti autografi, à l'occasion de son étude déjà citée consacrée à la tradition des Cinq chants, dont nous ne possédons pas de manuscrit autographe. Le choix de Segre, qui témoigne du profond développement des disciplines historico-linguistiques, part du principe que tous les usages de l'auteur étaient le fruit, en définitive, d'une «intention». En conséquence, dans tous les cas où les formes documentées ne correspondent pas aux usages avérés de l'Arioste, il faut attribuer cette déviance à l'intrusion du copiste et aux habitudes graphico-linguistiques de ce dernier. Le risque principal de cette méthode (que l'on pourrait contester également d'un point de vue général) est d'attribuer au poète non pas les formes dont on peut dire avec certitude qu'elles lui appartiennent, puisqu'elles sont écrites de sa propre main, et qui deviennent donc des formes vérifiées et utilisables pour de nouvelles comparaisons, mais celles que lui prête une reconstruction critique. Ainsi, dans plusieurs cas, Segre, dont l'approche consistait à analyser la correspondance ou non des formes écrites avec une certaine idée qu'il s'était faite de la langue de l'Arioste, a attribué au copiste des interventions de l'Arioste et réciproquement, si bien qu'il faudrait corriger l'apparat critique de son édition.

À partir de ces analyses fondées sur une hypothèse d'authenticité et d'uniformité linguistiques, Segre a fait un choix éditorial non moins innovant qu'inhabituel dans le panorama italien : il a corrigé, avec prudence et intelligence, certaines formes du manuscrit F qu'il jugeait non conformes à celles du poète. En résumé, Segre accepte les formes reconnues comme étant propres à l'Arioste même si ce dernier ne les utilisait plus au cours des années de la rédaction supposée du manuscrit $\mathrm{F}$; il maintient les formes qui y apparaissent en alternance

43. C. Bertani, «Sul testo e sulla cronologia delle Satire di Ludovico Ariosto», Giornale storico della letteratura italiana, 88, 1926, p. 256-281, puis 89, 1927, p. 1-36; M. Catalano, «Autografi e presunti autografi ariosteschi», Archivum Romanicum, 9, 1925, p. 37-66.

44. S. Debenedetti, «Intorno alle "Satire" dell' Ariosto», Giornale storico della letteratura italiana, 122, 1944-1946, p. $109-130$ 
avec d'autres formes plus récentes, mais corrige celles du copiste qui ne sont pas confirmées par les usages de l'auteur, usages qu'il reconstitue aussi en se fondant sur des éditions imprimées dont la variabilité linguistique est uniformisée sur la base des usages prédominants de l'époque (il remplace par exemple la préposition a lo par la forme contractée allo, modifie mattino en matino, popolo en populo) $)^{45}$. Dans la mesure où le modèle de langue qui lui sert de référence pour l'établissement des Satires est celui du Roland furieux, Segre s'appuie sur une autre hypothèse implicite, à savoir que les deux œuvres, de genres pourtant différents, partagent un même modèle linguistique.

Une telle méthode, si elle était instituée en norme pour l'édition de textes, modifierait sensiblement le profil linguistique des classiques italiens. Elle dénote une pleine confiance dans le fait que, y compris en matière de philologie des originaux, dès lors que l'originalité est partielle (comme dans le cas présent), «la reconstruction» puisse être considérée comme «plus véridique que le document», selon un aphorisme intelligemment provocateur de Contini qui, toutefois, l'avait énoncé pour la philologie des copies ${ }^{46}$. Cette méthode demande encore, du fait de son caractère innovant, à être discutée, notamment par les historiens de la langue. De manière générale, par-delà les menues corrections et intégrations qu'il conviendrait d'apporter à l'apparat critique, il faudrait réfléchir aux caractéristiques du manuscrit $\mathrm{F}$ et à l'opportunité d'une reconstruction et d'une présentation du texte qui parvienne à transformer l'état imparfait et inabouti des chefs-d'œuvre que le manuscrit nous livre - et qui est la seule trace avérée dont on dispose - en un livre cohérent, qui réponde mieux aux attentes d'un lecteur moderne.

Il serait possible de ménager un accès complet et facilité au manuscrit complexe des Satires à condition de résoudre préalablement deux problèmes. Il conviendrait en premier lieu de reconstruire la stratification des phases rédactionnelles dont témoigne le manuscrit $\mathrm{F}$ : on peut y parvenir en suivant notamment l'utilisation des encres différentes. Mais il serait nécessaire, pour que ce projet soit mené à son terme, de commencer par offrir une (ré)interprétation complète des textes en tenant compte de la biographie et du parcours intellectuel de l'auteur, ce qui n'est pas aisé au vu des maigres éléments dont on dispose (voir l'exemple tiré de la troisième satire - fig. 5). Il conviendrait, par ailleurs, d'envisager un nouvel apparat critique, qui permettrait au lecteur de distinguer les différents niveaux de la tradition manuscrite, autographe ou non (car nous avons trois témoins partiels), sans qu'il lui soit nécessaire, comme c'est actuellement le cas, de retracer depuis le début le travail de l'éditeur critique afin de s'orienter dans une suite de données indifférenciées.

Une nouvelle fois, les techniques d'analyse et les solutions éditoriales ne peuvent provenir que d'une prise en compte préliminaire de toutes les données, qui ménage un équilibre entre l'«intention» du texte et la perspective actualisante de l'interprète, dans un cercle herméneutique où conscience historique et conscience philologique s'appliquent pleinement ${ }^{47}$. Comme Contini l'a observé avec justesse, les éditeurs et les lecteurs sont confrontés à un «texte dans

45. L. Ariosto, Satire, op. cit., p. XXIII-XXIV.

46. G. Contini, «Filologia» (1977), rééd. dans Id., Breviario di ecdotica, Milan-Naples, Ricciardi, 1986, p. 3-66, ici p. 22.

47. Sur le texte et l'édition des Satires, voir S. Albonico, «Osservazioni paleografiche e considerazioni testuali sul manoscritto ferrarese delle "Satire" (ms. F)», dans M. Paoli et E. Russo (dir.), Paulina Spiechowicz (coll.), Studi sulle "Satire” di Ariosto, numéro monographique de L'Ellisse, 12, 2017, n² 2, p. 17-36. 


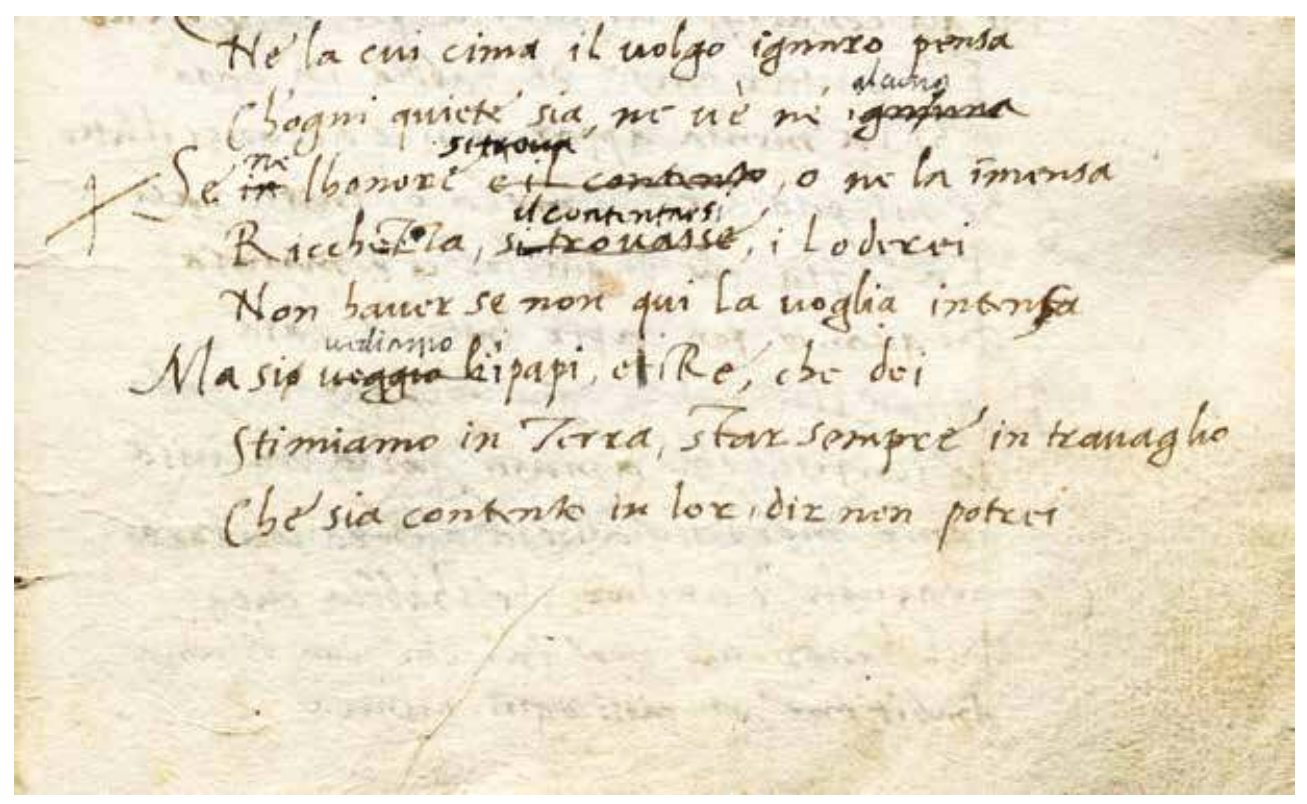

Fig. 5 : Ferrare, Biblioteca Comunale Ariostea, ms. Cl. I, B, It. 2, f. 19 rº, partie inférieure de la page (qui fait $220 \times 160 \mathrm{~mm}$ env.), v. 230-237 de la Satire III.

Selon C. Segre, les v. 232-233 ont subi une seule phase de correction :

1) Se in l'honore è il contento o ne la immensa I Ricchezza si trovasse, i' loderei ;

2) Se ne l'honore si trova o ne la immensa I Ricchezza il contentarsi, i' loderei.

La différence d'encres et de ductus est cependant évidente; de plus, l'analyse approfondie des leçons invite à supposer que la correction est le résultat de deux interventions distinctes. Il est probable que la première intervention, qui ne fut pas de nature purement grammaticale mais dérivait peut-être de la nécessité de supprimer le substantif «contento», ait eu pour effet de provoquer un remaniement de la syntaxe et de la distribution interne, mais avec une prosodie encore juste. La modification postérieure de in en $n e$, dictée par des exigences strictement grammaticales sur un vers déjà modifié peut expliquer le manque d'attention à la mesure du vers (de telles étourderies sont rares chez l'Arioste). La première intervention serait alors celle à l'encre foncée, et la seconde (qui conduit dans le vers précédent à transformer igniuna en ignuna, puis alcuna) celle à l'encre gris clair. Les trois étapes distinctes du remaniement seraient : 1) Se in l'honore è il contento o ne la immensa I Ricchezza si trovasse, i' loderei; 2) Se in l'honore si trova o ne la immensa Ricchezza il contentarsi, i' loderei; 3) Se ne l'honor>e< si trova o ne la immensa I Ricchezza il contentarsi, i' loderei.

(c) Ferrare, Biblioteca Comunale Ariostea.

Avec l'aimable autorisation du Ministero dei beni e delle attività culturali e del turismo. Reproduction interdite

le temps»; de même, toute édition doit être inscrite dans le contexte où elle a vu le jour, non pas tant pour en réduire l'importance que pour en saisir les implications et la valeur, et, par là, développer des hypothèses éditoriales et interprétatives nouvelles et différentes. Il est donc nécessaire, même dans le cas de l'Arioste, de revenir aux manuscrits, afin de renouveler un point de vue critique longtemps influencé par la renommée de certains spécialistes qui furent, en fin de compte, à l'origine du retard avec lequel nous travaillons aujourd'hui à mieux comprendre les caractéristiques littéraires et l'inscription historique de ces brouillons.

Traduit de l'italien par Pierre Musitelli avec la collaboration de Christian Del Vento

Simone Albonico, formé à l'université de Pavie où il a enseigné, est aujourd'hui professeur de Philologie et Littérature italienne à l'université de Lausanne. Ses travaux traitent de la littérature des XIve-xvie siècles (Dante, Pétrarque, Alberti, Sannazaro, Bembo, l'Arioste, Folengo, Della Casa) et portent une attention particulière au rapport aux classiques et à certains contextes (Milan entre le XIVe et le XVI ${ }^{\mathrm{e}}$ siècle, l'historiographie florentine). Il a publié, dans le domaine de la philologie d'auteur, des contributions sur l'Arioste, Della Casa et Manzoni. Il s'intéresse à la métrique italienne, à Leopardi, aux archives littéraires du Xxe siècle et aux solutions informatiques pour les disciplines humanistes. 


\section{Résumés \\ Pour un retour aux manuscrits de l'Arioste}

Santorre Debenedetti publia en 1937 ce que l'on nomme les «fragments autographes» du Roland furieux, qui documentent l'élaboration des octaves ajoutées par Ludovico Ariosto à la troisième édition de son chef d'œuvre (1532). Dans sa recension opportune de l'édition Debenedetti, le jeune Gianfranco Contini livra une interprétation des variantes de l'Arioste qui lui permettait de valider le jugement critique de Benedetto Croce, ce dernier discernant dans le Roland furieux la mise en œuvre d'un principe d'harmonie. Pour parvenir à cette conclusion, Contini ne prit en considération qu'une portion restreinte de l'élaboration textuelle du poème qui rentrait dans le champ de l'esthétique crocienne. Il manifesta par ailleurs son adhésion à d'autres postulats du philosophe napolitain, désormais remis en question depuis de longues années. La naissance de la «critique des variantes» d'inspiration continienne, fondée sur ces présupposés, a reçu au fil du temps un accueil plus ou moins favorable, mais elle n'a jamais fait l'objet d'une analyse historico-critique destinée à en vérifier le bien-fondé philologique et interprétatif. Le présent article entend amorcer une réflexion en ce sens.

Santorre Debenedetti published in 1937 what is known as the « olograph fragments » of Orlando Furioso, related to the drafting of the parts that were added by Ludovico Ariosto to the third edition of his masterpiece (1532). In his timely review of the Debenedetti edition, the young Gianfranco Contini interpreted Ariosto's variants in a way that allowed him to confirm the judgement of Benedetto Croce, who saw in Orlando Furioso the application of a principle of harmony. To reach such a conclusion, Contini considered only the part of the textual elaboration of the poem that was compatible with Crocean esthetics and endorsed other claims of the Neapolitan philosopher that are no longer considered valid. The birth of the Contini-inspired "critica delle varianti", based on those premises, has been greeted with varying degrees of enthusiasm, but it has never been reconsidered on a historico-critical basis, so as to verify its philological and interpretative relevance. The aim of this paper is to launch such a process of reconsideration.

Santorre Debenedetti veröffentlichte 1937 die sogenannten „handschriftlichen Fragmente“ von Orlando furioso, die die Ausarbeitung der Oktaven dokumentieren, die Ludovico Ariosto der dritten Ausgabe seines Meisterwerks (1532) hinzufügte. In seiner treffenden Rezension der Debenedetti-Ausgabe hat der junge Gianfranco Contini eine Interpretation der Varianten von Ariosto geliefert, die es ihm ermöglichte, das kritische Urteil von Benedetto Croce zu bestätigen, der in Orlando furioso die Umsetzung eines Prinzips der Harmonie erkannte. Um zu dieser Schlussfolgerung zu gelangen, berücksichtigte Contini nur einen begrenzten Teil der textuellen Ausarbeitung des Gedichts, das in den Bereich der Ästhetik von Croce fällt. Außerdem bekundete er sein Festhalten auch an anderen Postulaten des neapolitanischen Philosophen, was seit vielen Jahren in Frage gestellt wurde. Basierend auf diesen Annahmen entstand eine „,ritique des variantes“, die durch Contini inspiriert wurde. Sie hat im Laufe der Zeit eine mehr oder weniger günstige Rezeption erfahren, war jedoch nie Gegenstand einer historisch-kritischen Analyse, die sich ihrer philologischen und interpretativen Verdienste widmet. Der vorliegende Artikel beabsichtigt eine Reflexion in diese Richtung anzustoßen.

En 1937, Santorre Debenedetti publicó los denominados "fragmentos autógrafos" del Orlando furioso, que muestran la elaboración de las octavas añadidas por Ludovico Ariosto a la tercera edición de su obra maestra (1532). En su pertinente recensión de la edición Debenedetti, el joven Gianfranco Contini elaboró una interpretación de las variantes de Ariosto tendiente a confirmar el juicio crítico de Benedetto Croce, quien veía en el Orlando furioso la realización de un principio de armonía. Para llegar a esa conclusión, Contini aisló un sector muy restringido de la elaboración textual del poema, compatible con el campo de la estética de Croce, y adhirió, asimismo, a otros postulados del filósofo napolitano, puestos en tela de juicio desde hace años. El surgimiento de la "crítica de las variantes" continiana, basada en estos presupuestos, fue aceptada más o menos favorablemente a lo largo de los años, pero no ha sido nunca objeto de un análisis histórico-crítico destinado a verificar sus fundamentos filológicos e interpretativos. El presente artículo intenta iniciar una reflexión en este sentido.

Em 1937, Santorre Debenedetti publicou o que se chama de «fragmentos de autógrafos », de Orlando furioso, que documenta a elaboração das partes extras adicionadas por Ludovico Ariosto na terceira edição da sua obra-prima (1532). Na sua oportuna revisão da edição Debenedetti, o jovem Gianfranco Contini interpretou as variantes de Ariosto de tal maneira que lhe permitisse validar o julgamento crítico de Benedetto Croce, que via em Orlando furioso, a execução de um princípio de harmonia. Para chegar à esta conclusão, Contini apenas considerou uma parcela muito restrita do texto do poema compatível com o limite da estética crociana. Por outro lado, ele manifestou sua adesão à outros postulados do filósofo napolitano, tendo sido por muito tempo, questionados. O nascimento da « crítica das variantes » de inspiração continiana, fundada em seus pressupostos, teve, ao longo do tempo, uma recepção mais ou menos favorável, porém, nunca foi objeto de uma análise histórico-crítica destinada à verificar a solidez filológica e interpretativa. O presente artigo procura iniciar uma reflexão nesse sentido.

Nel 1937 Santorre Debenedetti pubblicò i cosiddetti "frammenti autografi" dell' Orlando furioso, relativi all'elaborazione delle parti aggiunte da Ludovico Ariosto alla terza edizione del suo capolavoro (1532). Recensendo tempestivamente l'edizione Debenedetti, il giovane Gianfranco Contini interpretò le varianti ariostesche in modo tale da ricavarne conferme alla formula critica di Benedetto Croce che vedeva nel Furioso la realizzazione di un principio di armonia. Per arrivare a questa conclusione Contini isolò un settore molto ristretto dell'elaborazione testuale ariostesca compatibile con i limiti dell'estetica crociana, e aderì ad altri assunti del filosofo napoletano considerati ormai da tempo irricevibili. La nascita della "critica delle varianti" continiana su questi presupposti è stata variamente celebrata nel tempo ma mai riconsiderata in termini storico-critici puntuali, per verificarne la tenuta filologica e interpretativa. Il contributo avvia una riflessione in questa direzione. 